\title{
ESA THESEUS and cataclysmic variables
}

\author{
René Hudec ${ }^{1,2,3 *}$ \\ ${ }^{1}$ Astronomical Institute of the Academy of Sciences of the Czech Republic \\ Fricova 298 - CZ 25165 Ondrejov, Czech Republic \\ ${ }^{2}$ Czech Technical University in Prague, Faculty of Electrical Engineering, \\ Technicka 2, CZ 16000 Prague, Czech Republic \\ ${ }^{3}$ Kazan Federal University, Kazan, Russian Federation \\ E-mail: rene.hudec@gmail.com
}

\section{Vojtech Simon ${ }^{1,2}$}

${ }^{1}$ Astronomical Institute of the Academy of Sciences of the Czech Republic Fricova 298 - CZ 25165 Ondrejov, Czech Republic

${ }^{2}$ Czech Technical University in Prague, Faculty of Electrical Engineering, Technicka 2, CZ 16000 Prague, Czech Republic

\section{Lorenzo Amati ${ }^{3}$}

${ }^{3}$ INAF, Bologna, Italy

\section{Filippo Frontera ${ }^{3,4}$}

${ }^{4}$ University Ferrara, Ferrara, Italy

\section{Enrico Bozzo 5}

${ }^{5}$ University of Geneva, Geneva, Switzerland

\section{Paul T. O’Brien ${ }^{6}$}

${ }^{6}$ University of Leicester, Leicester, Great Britain

\section{Diego Goetz ${ }^{7}$}

${ }^{7}$ CEA, Toulouse, France

The capabilities of the considered space mission THESEUS for investigation of cataclysmic variables (CVs) are discussed.Transient High-Energy Sky and Early Universe Surveyor (THESEUS) is a space mission proposal accepted by the European Space Agency for a phase A study that would study gamma-ray bursts and X-rays for investigating the early universe and for the multimessenger astrophysics. It involves a Lobster-Eye X-ray telescope as well. The THESEUS and SMILE international consortia involve the Czech Technical University in Prague and the Czech teams are expected to contribute to the project, mainly to the X-ray telescope and related science and software.

The Golden Age of Cataclysmic Variables and Related Objects V (GOLDEN2019)

2-7 September 2019

Palermo, Italy

* Speaker. 


\section{Introduction}

THESEUS is a mission concept proposed in response to the ESA call for medium-size missions (M5) within the Cosmic Vision Programme and selected by ESA on 2018 May 7 to enter an assessment phase study. The mission is designed to vastly increase the discovery space of the high energy transient phenomena over the entirety of cosmic history (Amati et al. 2018, Stratta et al. 2018). Its primary scientific goals will address the Early Universe ESA Cosmic Vision themes "How did the Universe originate and what is made of" (4.1, 4.2 and 4.3) and will also impact on "The gravitational wave Universe" (3.2) and "The hot and energetic Universe themes". This is achieved via a unique payload providing an unprecedented combination of: 1) wide and deep sky monitoring in a broad energy band $(0.3 \mathrm{keV}-20 \mathrm{MeV}) ; 2)$ focusing capabilities in the soft X-ray band providing large grasp and high angular resolution; and 3) on board near-IR capabilities for immediate transient identification and redshift determination. (https://www.isdc.unige.ch/theseus/).

\section{ESA THESEUS}

The main scientific goals of the proposed mission are to explore the Early Universe (cosmic dawn and reionization era) by unveiling a complete census of the Gamma-Ray Burst (GRB) population in the first billion years and to perform an unprecedented deep monitoring of the X-ray transient Universe (Frontera et al. 2018, O'Brien et al. 2018). The foreseen payload of THESEUS includes the following instrumentation (https://www.isdc.unige.ch/theseus/): Soft X-ray Imager (SXI, 0.3-6 keV): a set of 4 lobster-eye telescopes units, covering a total field of view (FOV) of $1 \mathrm{sr}$ with source location accuracy < 1-2 arcmin; InfraRed Telescope (IRT, 0.7-1.8 microns): a $0.7 \mathrm{~m}$ class IR telescope with $10 \times 10$ arcmin FOV, for fast response, with both imaging and spectroscopy capabilities; X-Gamma rays Imaging Spectrometer (XGIS, $2 \mathrm{keV-20} \mathrm{MeV):} \mathrm{a} \mathrm{set} \mathrm{of} \mathrm{coded-mask}$ cameras using monolithic X-gamma rays detectors based on bars of Silicon drift detectors coupled with CsI crystal scintillator, granting a $1.5 \mathrm{sr}$ FOV, a source location accuracy (90\% C.L.) better than 8 arcmin in 2-150 keV energy band and an unprecedently broad energy band. The mission profile includes: an onboard data handling units (DHUs) system capable of detecting, identifying and localizing likely transients in the SXI and XGIS FOV; the capability of promptly (within a few tens of seconds at most) transmitting to ground the trigger time and position of GRBs (and other transients of interest); and a spacecraft slewing capability of $10-20$ degrees $/ \mathrm{min}$ ). The baseline launcher / orbit configuration is a launch with Vega-C to a low inclination low Earth's orbit (LEO, $600 \mathrm{~km},<5$ degrees), which has the unique advantages of granting a low and stable background level in the high-energy instruments, allowing the exploitation of the Earth's magnetic field for spacecraft fast slewing and facilitating the prompt transmission of transient triggers and positions to the ground.

For more details on ESA project THESEUS please see Amati et al. (2018) and Stratta et al. (2018).

\section{Czech participation in THESEUS}

CTU in Prague and Rene Hudec are the THESEUS project consortium members. The Czech participation is based on very long experience with imaging X-ray telescopes and monitors in the 
Czech Republic with emphasis on wide field X-ray monitors Lobster Eye type. The expected Czech contribution is as follows: SXI, optics tube, mechanics, electronics, and software. There is expected participation of Czech space industry. The Czech participation in the project is supported by the Czech PRODEX Office for phases A and 0.

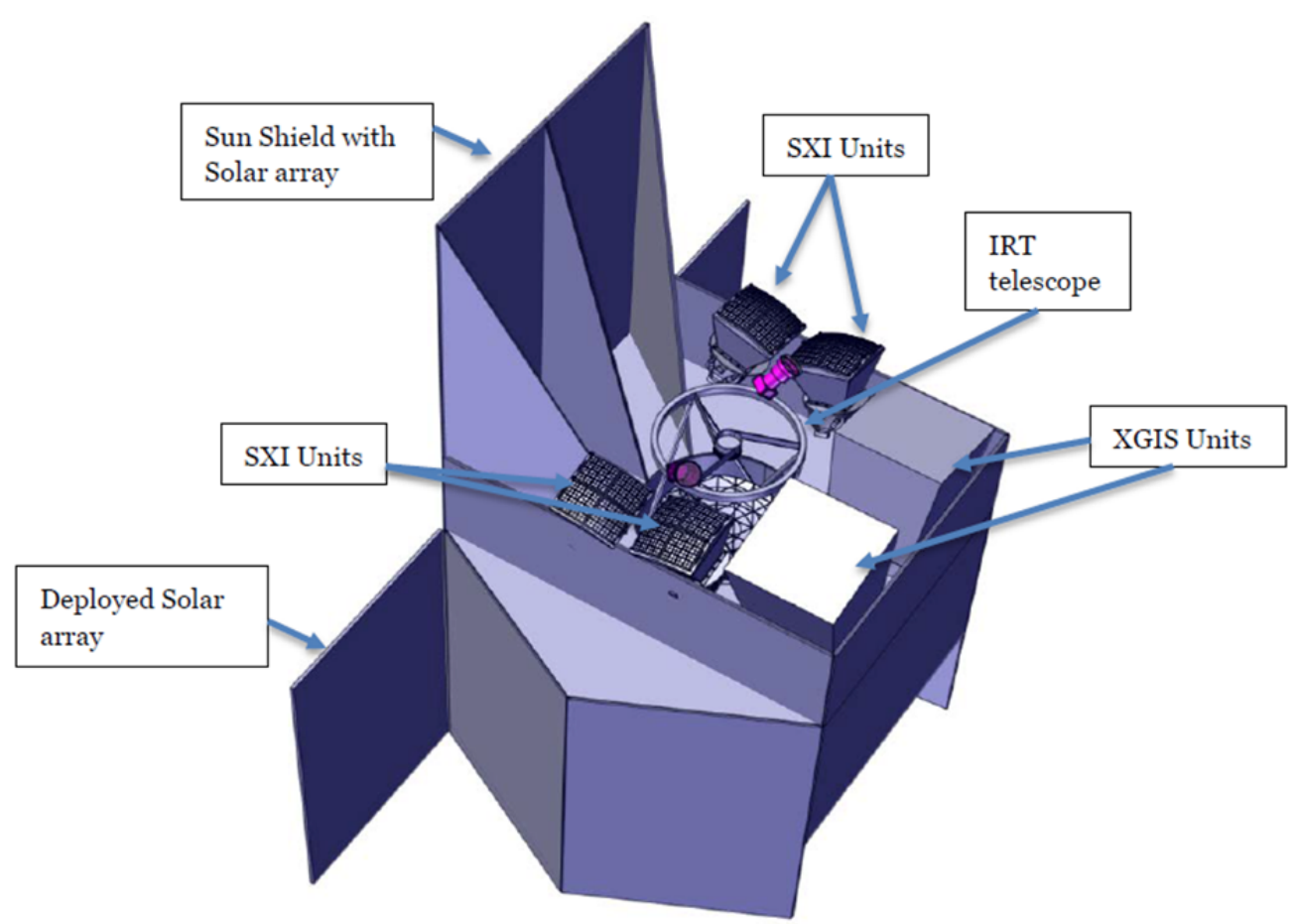

Figure 1: THESEUS ESA study model at the time of the conference. Note that the current (October 2020) baseline payload configuration includes two SXI cameras only (for reasons of minimizing technical and programmatic risks).

\section{Cataclysmic variables with THESEUS}

The telescopes onboard THESEUS can be used also for observing cataclysmic variables (CV) (e.g., Warner 1995) and symbiotic binaries (e.g., Kenyon 1986). Especially the hard X-ray imaging spectrometer THESEUS/XGIS will be able to detect and observe these objects over a very wide range of energies. It will be able to detect the contribution of various emitting components of the object.

The strength of the magnetic field of the white dwarf (WD) plays a big role in influencing the structure of the accreting matter (e.g., Warner 1995). It also influences the spectrum and the detectability of the object in various X-ray bands. Magnetic CVs, in which mass is accreted onto the polar caps in the vicinity of the magnetic pole(s) of the WD (not onto boundary layer encircling its equator) emit radiation over a very broad range of energies, reaching to very hard X-rays. This considers polars and intermediate polars. 


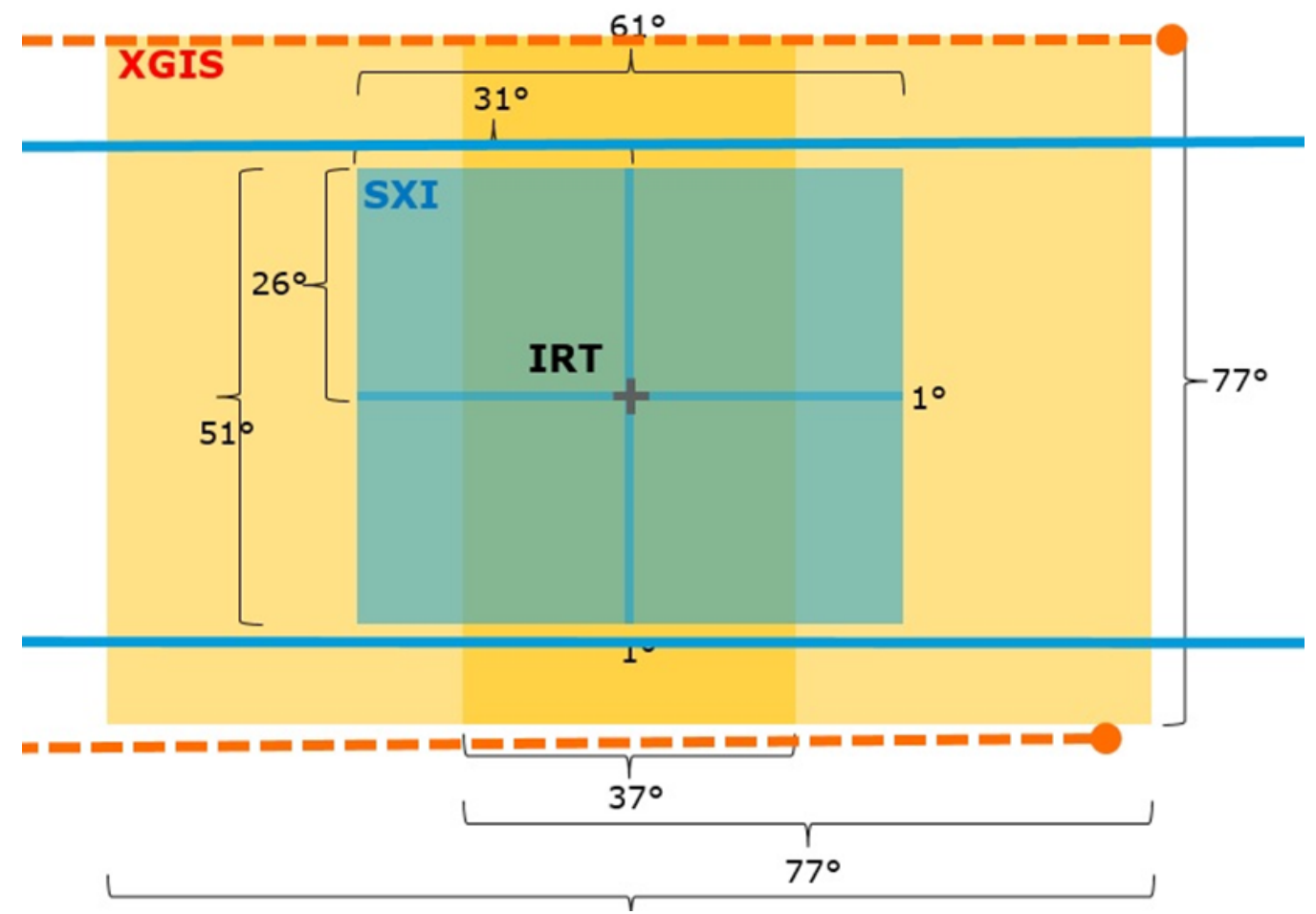

Figure 2: FOV of THESEUS payload at the time of the conference. Note that the current (October 2020) baseline payload configuration includes two SXI cameras only (for reasons of minimizing technical and programmatic risks).

\subsection{Symbiotic binaries}

Symbiotic binaries contain the compact object with a late-type companion in a very wide orbit (years). This companion is a giant and mass accretion onto the compact object often occurs via stellar wind, not via lobe overflow. The reason is that the donor star does not fill its Roche lobe. The accretion disk may sometimes be present. The compact object is the white dwarf (WD) in most cases but the neutron star (NS) can be present in some cases. A very hard X-ray emission can be produced in some symbiotics with the WD (e.g., RT Cru (Luna \& Sokoloski 2007)) and NS accretors (GX1+4 (Nagase 1989)).

We show the X-ray observations of the long-term activity of GX1+4 as an example (Fig. 3). GX1+4 is a NS symbiotic with the orbital period of about a year (Nagase 1989). Its optical counterpart is V2116 Oph (Glass \& Feast 1973).

Figure 3 shows the long-term activity of GX1+4 over 2000 days. We compare the light curves from the X-ray monitors ISS/MAXI (Matsuoka et al. 2009) and Swift/BAT (Krimm et al. 2014). This combination of the light curves shows the differences between the features of activity in the individual energy bands. GX1+4 displays flares in the observed time segment. The strengths of some of these features differ for the individual X-ray energy bands.

This combined energy band also suggests what we can expect from XGIS onboard THESEUS, planned to observe in a very wide energy band. Because of the wide field of view of XGIS, even 
the observations of such sources by THESEUS will bring important pieces of information about their long-term activity.
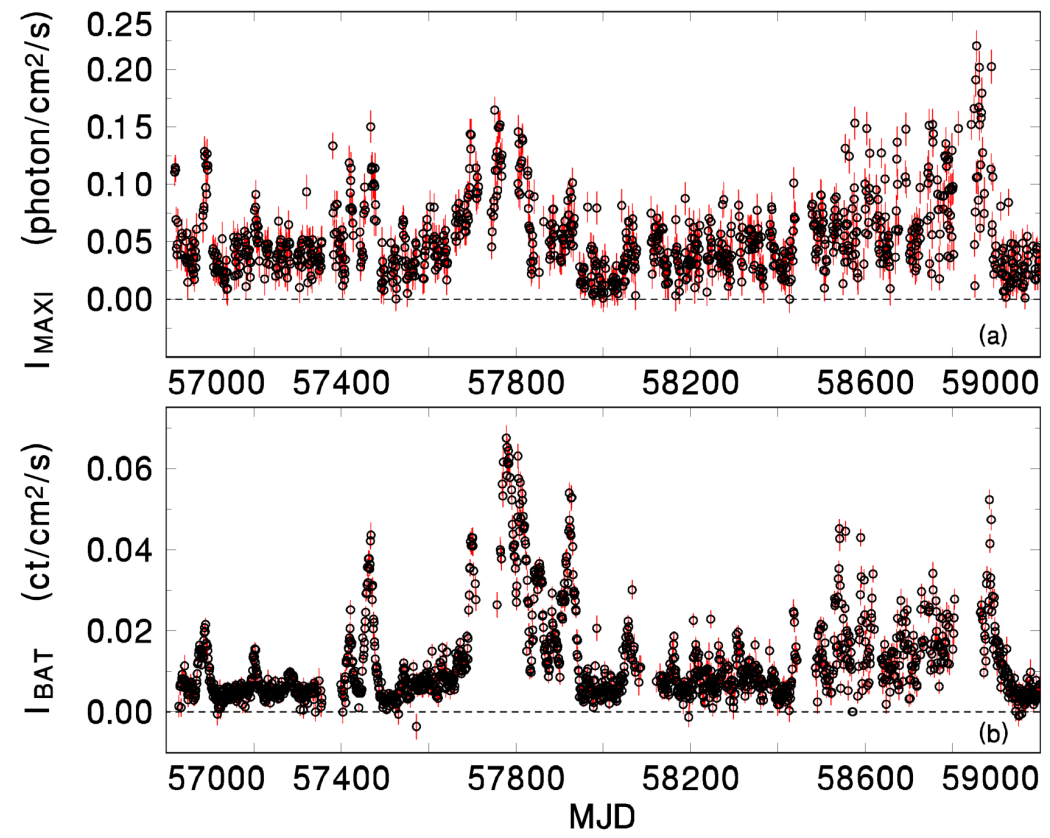

Figure 3: The long-term activity of GX1+4. The light curves of the data from the X-ray monitors ISS/MAXI $(2-20 \mathrm{keV})$ (Matsuoka et al. 2009) and Swift/BAT (15-50 keV) (Krimm et al. 2014) are used.

\section{Conclusions}

The THESEUS mission is designed to vastly increase the discovery space of the high energy transient phenomena over the entirety of cosmic history. This mission represents a scientifically valuable satellite project with wide international participation, including Czech scientists. The satellite design includes a Lobster Eye (LE) X-ray telescope. Missions based on LOBSTER EYE telescopes, if approved for space flight, will significantly contribute to the CVs science.

\section{Acknowledgements}

The author acknowledges continuous support by the Astronomical Institute of the Czech Academy of Sciences in Ondrejov under Institutional project RVO 67985815 as well as grants GA CR 13-33324S and Mobility MSMT CZ-A project 7AMB18AT036. We also acknowledge the H2020 project AHEAD funded by the European Union as Research and Innovation Action under Grants No: 654215 and 871158.

\section{References}

[1] Amati, L., O’Brien, P., Götz, D., et al. 2018, Advances in Space Research, 62, 191. doi:10.1016/j.asr.2018.03.010

[2] Stratta, G., Ciolfi, R., Amati, L., et al. 2018, Advances in Space Research, 62, 662. doi:10.1016/j.asr.2018.04.013 
[3] Stratta, G., Amati, L., Ciolfi, R., et al. 2018, , 89, 205

[4] Frontera, F., Amati, L., O’Brien, P., et al. 2018, , 89, 157

[5] O’Brien, P., Bozzo, E., Willingale, R., et al. 2018, , 89, 130

[6] Hudec, R., et al., Proc. SPIE 5488, UV and Gamma-Ray Space Telescope Systems, (11 October 2004); doi: 10.1117/12.551915, 2004

[7] Hudec, R., Pína, L., Inneman, A., Švéda, L., LOBSTER - Astrophysics with Lobster Eye Telescopes, in Exploring the Cosmic Frontier, ESO Astrophysics Symposia European Southern Observatory 2007, pp.73-74, 2007

[8] Inneman, A., et al., 2000, Proc. SPIE, 4138, 94

[9] Hudec, R., et al., 2000, SPIE Proc. 4012, 432

[10] Hudec, R., et al., 2003, SPIE Proc. 4851, 578

[11] Hudec, R., et al., 2004a, SPIE Proc. 5488, 449

[12] Hudec, R., et al., 2004b, Nucl. Phys. B Proc. Suppl. 132, 320

[13] Glass, I. S., \& Feast, M. W., 1973, NPhS, 245, 39

[14] Kenyon, S. J., 1986, The symbiotic stars, Cambridge: University Press

[15] Krimm, H. A., et al., 2013, ApJS, 209, 14

[16] Luna, G. J. M., \& Sokoloski, J. L., 2007, ApJ, 671, 741

[17] Matsuoka, M., et al., 2009, PASJ, 61, 999

[18] Nagase, Fumiaki, 1989, PASJ, 41, 1

[19] Warner, B., 1995, Cataclysmic Variable Stars, Cambridge Univ. Press, Cambridge 